\title{
A ANÁliSE dE CONTEÚdo E SUA IMPORTÂNCIA COMO INSTRUMENTO DE INTERPRETAÇÃO DOS DADOS QUALITATIVOS NAS PESQUISAS EDUCACIONAIS
}

\author{
CONTENT ANALYSIS AND ITS IMPORTANCE AS AN INSTRUMENT FOR THE \\ INTERPRETATION OF QUALITATIVE DATA IN EDUCATIONAL RESEARCH
}

Lana Lisiêr de Lima Palmeira Doutora em Educação pela Universidade Federal de Alagoas - UFAL Professora da UFAL em Arapiraca - AL - Brasil. lanallpalmeira@outlook.com

(D) Carla Priscilla Barbosa Santos Cordeiro Doutora em Educação pela Universidade Federal de Alagoas - UFAL Professora do Cesmac em Arapiraca - AL - Brasil. carlapriscilla.bsc@gmail.com

Edna Cristina do Prado Pós-doutorado em Educação no Instituto de Educação da Universidade de Lisboa - IE/UL Professora da UFAL em Maceió - AL - Brasil. wiledna@uol.com.br

Resumo: O presente artigo pretende demonstrar a relevância da Análise de Conteúdo como técnica de interpretação de dados nas pesquisas de forma geral e, mais especificamente, na área educacional. Realizou-se, assim, uma pesquisa exploratória sobre a temática, com uma revisão sistemática dos estudos que se valeram do método na área educacional entre 2015 e 2019. Os dados foram colhidos por meio de um levantamento bibliográfico em três bases de dados: Scielo, Portal da Capes de periódicos e Biblioteca Digital Brasileira de Teses e Dissertações (BDTD). Seguindo-se este percurso, foi possível traçar um panorama da importância da técnica e de seu uso ao longo do período analisado. Com isto, evidenciou-se a importância da análise de conteúdo em pesquisas educacionais para análise de dados qualitativos.

Palavras-chave: Análise de conteúdo. Técnica de pesquisa. Área educacional.

Abstract: The present article aims to discuss the exercise of autonomy among education professionals in the construction of meaning vis-à-vis proposals that are formulated at a macro level inside governmental structures. To that end, in light of Foucaultian assumptions, I begin with the contextualization of educational issues in our society of performance as whole, seeking to understand the multiple interferences of neoliberal economics in education, as well as to understand how the subject is constituted in the face of power relations as they pertain to of "conducting the actions of others". Such assumptions are amplified by Basil Bernstein, who seeks to understand how power and control mechanisms interfere in the relationship among subjects within the same or different hierarchical levels, generating $\backslash$ the reproduction/production of certain messages from specific voices. Finally, it may be concluded that the exercise of autonomy and the conscious use of reason are possible when embedded in a rational, theoretical and ethical stance regarding major current issues.

Keywords: Content analysis. Research technique. Educational area.

\section{Para citar - ABNT NBR 6023:2018}

PALMEIRA, Lana Lisiêr de Lima; CORDEIRO, Carla Priscilla Barbosa Santos; PRADO, Edna Cristina do. A análise de conteúdo e sua importância como instrumento de interpretação dos dados qualitativos nas pesquisas educacionais. Cadernos de Pós-graduação, São Paulo, v. 19, n. 1, p. 14-31, jan./jun. 2020. Disponível em: https://doi.org/10.5585/cpg.v19n1.17159. 


\section{Introdução}

A análise de conteúdo é uma técnica de análise das comunicações que irá examinar detalhadamente o que foi coletado na pesquisa, sem deixar de considerar as observações do pesquisador ao longo do caminho trilhado para a obtenção desses achados. (BARDIN, 2016).

Essa técnica de análise tem suas origens ligadas a Laswell que, em meados de 1915, utilizoua nos Estados Unidos, realizando análise de imprensa e de propagandas. (BARDIN, 2016).

Gradativamente, a análise de conteúdo foi ganhando espaço entre as mais diversas áreas, tais como a linguística e a psiquiatria, mostrando-se um instrumento de sucesso nas pesquisas que lançaram mão da mesma, o que fez com que cada vez mais fosse havendo sua difusão entre os demais campos do conhecimento. (BARDIN, 2016).

A definição da análise de conteúdo pode ser concebida de várias maneiras, seja levando em conta a ancoragem teórica e a subjetividade do pesquisador, seja apropriando-se de definições de cunho semântico das mensagens, ou mesmo buscando a inferência, que nada mais é que "uma operação lógica pela qual se admite uma proposição em virtude da sua ligação com outras proposições já aceitas como verdadeiras". (BARDIN, 2016, p.45).

Deve-se ressaltar também a conotação social da análise de conteúdo, pois existem dimensões na investigação social que descrevem o processo de pesquisa no que diz respeito à combinação de elementos. Além disso, deve-se considerar que a análise de conteúdo é uma técnica que busca produzir inferências de uma mensagem para todo um contexto no qual a pesquisa se insere de forma objetiva (BAUER; GASKELL, 2003).

Levando-se em consideração estas premissas, surgiu o problema de estudo desta pesquisa: Qual a relevância da Análise de Conteúdo como técnica de interpretação de dados nas pesquisas de forma geral e, mais especificamente, na área educacional?

Assim, tomando por base a importância da análise de conteúdo para a produção de novas descobertas no campo do conhecimento científico, desenvolver-se-á no presente artigo um estudo que, de início, mostrará a evolução do método científico na vertente histórico-filosófica, passando posteriormente a trabalhar os conceitos fundantes da análise do conteúdo, bem como suas fases de desenvolvimento no prisma da operacionalização da técnica, ressaltando sua relevância em especial para as pesquisas do campo educacional realizadas no Brasil nos últimos anos. Afinal, como assegura Bardin (2016), citando Lasswell, Lerner e Pool (1952), “a análise de conteúdo deve começar onde os modos tradicionais de investigação acabam".

Partiu-se de uma pesquisa exploratória sobre a utilização da análise de conteúdo em pesquisas educacionais entre 2015 e 2019. Foi realizada uma revisão sistemática dos estudos que utilizaram a análise de conteúdo nos últimos anos, por meio de um levantamento bibliográfico em 
três bases de dados selecionadas para a análise: Scielo, Portal da Capes de periódicos e Biblioteca Digital Brasileira de Teses e Dissertações (BDTD). Com isto, tornou-se possível compreender a evolução quantitativa da utilização da análise de conteúdo nas pesquisas educacionais no país.

\title{
O método científico: um olhar acerca de sua evolução histórico-filosófica
}

Sabe-se que a pesquisa é, de maneira geral, um conjunto de exercícios de investigação que obedece a uma série de critérios/métodos em busca de novos conhecimentos científicos.

Assim, para melhor embasar as ideias que nortearão o presente trabalho, busca-se fazer um breve resgate histórico-filosófico em torno da ciência e do método científico, evidenciando a forma como a ciência ganhou forma no mundo da pesquisa.

Desse modo, fundamental, de início, trazer à tona a origem etimológica das expressões "método" e "ciência".

De acordo com o que ensina Abbagnano (2000, p.668), a palavra método, do latim "methodus":

\begin{abstract}
tem dois significados fundamentais: $1^{\circ}$ qualquer pesquisa ou orientação de pesquisa e o $2^{\circ}$ uma técnica particular de pesquisa. No primeiro significado não se distingue de investigação ou doutrina, $\mathrm{O}$ segundo significado é mais restrito e indica um procedimento de investigação organizado, repetível e autocorrigível, que garanta a obtenção de resultados válidos.
\end{abstract}

Vê-se, assim, que a segunda acepção é onde há de fato uma ordenação de regras, ou seja, onde existe uma construção conceitual e procedimental para se atingir realmente a ideia de método, sendo este o que mais vai ancorar o ideal científico.

Aqui, merece ser ressaltado que tal construção é algo particular de um período que se busca trilhar nessas linhas inaugurais do presente trabalho, a fim de demonstrar como o método plasmouse na ciência moderna e como esse método, de certa forma, influenciou toda a epistemologia.

Analisando o conceito da expressão ciência, com origem inglesa calcada na expressão "Science of Science", tem-se que ela representa "a exposição de modo sistemático do princípio fundamental em que se apoiam as outras ciências. Qualquer ciência possível tem um princípio fundamental que nela não pode ser demonstrado, mas que já deve ter sido verificado antes dela." (ABBAGNANO, 2000, p.140).

Significa dizer que a experimentação é um modo sistemático que, para se tornar empiria necessita de uma teoria. Ou seja, não existe ciência sem sistema, nem sistema sem teoria, sendo, por isso, fundamental a correlação na ciência entre teoria e prática. (POPPER, 1985).

Feita essa contextualização, importante analisar, ainda dentro dessa vertente, a imagem do universo na Revolução Científica, lembrando que os exemplos mais evidentes dessas revoluções 
são os famosos episódios de desenvolvimento científico que já no passado foram frequentemente indicados como reviravoltas fundamentais que se ligam aos nomes de Copérnico, de Newton, de Lavoisier e de Einstein.

Convém ainda ressaltar que durante os 150 anos que decorrem entre Copérnico e Newton, não é apenas a imagem do mundo que se transforma. Vinculada a essa transformação, está o desenvolvimento da mudança das ideias sobre o próprio homem, sobre a ciência, sobre o homem da ciência, sobre o trabalho científico, sobre as relações entre ciência e sociedade, ciência e filosofia e até mesmo entre saber científico e fé religiosa.

É justamente a partir desse rompimento que se desenvolve hoje a ideia que temos de ciência moderna, sendo essa perspectiva de ciência moderna, de experimentação e observação uma concepção distinta da ideia que se tinha da ciência antes da modernidade.

Isso vai de certa forma desaguar no conceito de paradigma em Thomas Khun, quando ele afirma que a revolução científica configura-se como um poderoso movimento de ideias que adquire, no século XVII, suas características determinantes na obra de Galileu, de Bacon e Descartes e que depois encontrará a sua expressão na imagem newtoniana do universo concebido como máquina, ou seja, como relógio, destacando ainda que isso comprova a mudança operada na forma com que os homens concebem a si e ao mundo, ou seja“(...) quando os paradigmas mudam, o próprio mundo toma novas direções” (KHUN, 1962, p. 17)

O fato mais importante dessa ruptura de paradigmas é que, com elas, os cientistas passam a enxergar horizontes diversos, deixando de olhar o mundo com instrumentos tradicionais e nas mesmas direções de antes. A transição do paradigma do medievo para a modernidade, especificamente no caso da revolução científica, é uma reconstrução do campo sobre novas bases, é uma reconstrução da própria ideia do homem e da humanidade. (KHUN, 1962).

Assim, nessa fase de mudanças, a ciência torna-se saber experimental, não mais sendo a intuição privilegiada do mago ou astrólogo iluminado, individualmente, nem o comentário de um filósofo que dizia ser detentor da verdade, isto é, a ciência deixa de ser um discurso sobre o "mundo de papel" e passa a ser uma investigação sobre o "mundo da natureza" como afirma Galileu (REALE, 2003).

Além disso, tem-se que, em sua essência, a ciência é pública - e o é por questões de métodos, ou seja, é a ideia de ciência metodologicamente regulada e publicamente controlável que exige o surgimento das novas instituições científicas, como as academias, os laboratórios, dentre outros.

Então, a revolução científica é precisamente aquele processo histórico do qual decorre a ciência experimental, vale dizer, uma nova forma de saber, nova e diferente do saber religioso, 
PALMEIRA, Lana Lisiêr de Lima; CORDEIRO, Carla Priscilla Barbosa Santos; PRADO, Edna Cristina do. A análise de conteúdo e sua importância como instrumento de interpretação dos dados qualitativos nas pesquisas educacionais

metafísico, astrológico e também técnico e arsenal - o que, como já foi dito, possibilita o surgimento da comunidade científica.

Por outro lado, não é excessivo evidenciar que o olhar contemporâneo da Filosofia, a exemplo de Heidegger em sua obra "Ser e Tempo" vai questionar essa concepção tecnicista da ciência, quando vem nos falar principalmente do esquecimento do ser ou de como ele fala do $\operatorname{Dasein}^{2}(2014$, p.39)

Isso também vai ser endossado por Gadamer em sua obra "Verdade e Método", quando ele vai fazer um olhar hermenêutico sobre a ciência, deixando de ter uma visão cíclica sobre o processo de construção do conhecimento, defendendo que o saber verdadeiro não nasce única e exclusivamente da aplicação de um procedimento metodológico, mas se constrói utilizando uma relação de dependência a pressupostos dos quais esse saber precisa não só tomar consciência como reconhecer momentos peculiares de seu processo (GADAMER, 1997).

Afinal, como afirmava Gadamer, só se pode aprender através do diálogo. É o diálogo que permite a humanização, a participação ativa de todos no processo em que está inserido (1997, p.177).

Dentro desse raciocínio, não seria despiciendo trazer à reflexão o que ensina Hannah Arendt, em sua obra "A Condição Humana", quando ressalta:

\begin{abstract}
Esse homem futuro, que os cientistas nos dizem que produzirão em menos de um século, parece imbuído por uma rebelião contra a existência humana tal como ela tem sido dada - um dom gratuito vindo de lugar nenhum (secularmente falando) que ele deseja trocar, por assim dizer, por algo produzido por ele mesmo. Não há razão para duvidar de que sejamos capazes de realizar tal troca, assim como não há motivo para duvidar de nossa atual capacidade de destruir toda vida orgânica na Terra. A questão é apenas se desejamos usar nessa direção nosso novo conhecimento científico e técnico, e essa questão não pode ser decidida por meios científicos; é uma questão política de primeira grandeza, cuja decisão, portanto, não pode ser deixada a cientistas profissionais ou a políticos profissionais. (2016, p.03).
\end{abstract}

Neste contexto de evolução do conceito de ciência e da própria metodologia científica, surge e se desenvolve a análise de conteúdo como uma nova forma de interpretar resultados de dados colhidos em pesquisas empíricas, permitindo que a racionalidade inerente às ciências exatas pudesse ser apropriada pelas ciências humanas. Como bem esclarece Moraes (1999, p. 8):

A análise de conteúdo tem sua origem no final do século passado. Suas características e diferentes abordagens, entretanto, foram desenvolvidas, especialmente, ao longo dos últimos cinquenta anos.

Mesmo tendo sido uma fase de grande produtividade aquela em que esteve orientada pelo paradigma positivista, valorizando sobremodo a objetividade e a quantificação, esta metodologia de análise de dados está atingindo novas e mais desafiadoras possibilidades na medida em que se integra cada vez mais na exploração qualitativa de mensagens e informações. Neste sentido, ainda que eventualmente não com a denominação de análise de conteúdo, se insinua em trabalhos de natureza dialética, fenomenológica e etnográfica, além de outras. 
Doutro lado, é preciso reconhecer que, mesmo tendo a análise de conteúdo iniciado sua trajetória impregnada pela dureza metodológica inerente ao positivismo ao longo da sua evolução, essa técnica de análise conseguiu romper com o rigor típico das ciências exatas, como esclarece Moraes (1999, p. 10):

\footnotetext{
$\mathrm{Na}$ sua evolução, a análise de conteúdo tem oscilado entre o rigor da suposta objetividade dos números e a fecundidade sempre questionada da subjetividade. Entretanto, ao longo do tempo, têm sido cada vez mais valorizadas as abordagens qualitativas, utilizando especialmente a indução e a intuição como estratégias para atingir níveis de compreensão mais aprofundados dos fenômenos que se propõe a investigar.
}

Este método, a partir da perspectiva acima mostrada, conseguiu olhar para a pesquisa científica não como um processo de engessamento do conhecimento, mas sim como um elemento que, respeitando os critérios exigidos, procure valorar técnicas dotadas de capacidade de avaliar, de fato, determinada realidade, dentro dos padrões que norteiam o universo investigado.

Dessa forma, é possível compreender que a análise de conteúdo evoluiu enquanto método de análise de dados ao longo do tempo. Por ser uma técnica que leva em consideração inúmeros elementos que circundam os horizontes pesquisados, desprende-se, de certa forma, dessa "dureza metodológica" demonstrada anteriormente, captando com muita riqueza detalhes e circunstâncias que envolvem determinado objeto, esboçando, na leitura dos achados da pesquisa, um retrato mais fiel do que os dados revelam, conforme se verá a seguir.

\section{O que é a análise de conteúdo?}

Após trabalhar a evolução da ciência, tentando abordar o que representa a pesquisa e detalhando o que ela vem a ser dentro da evolução histórico-filosófica, convém agora voltar a atenção ao que significa a Análise de Conteúdo, objeto central deste estudo.

Sabe-se que toda pesquisa necessita de uma definição quanto ao critério para a interpretação dos seus achados, sendo uma das fases mais decisivas para o pesquisador a escolha adequada não só das técnicas de coleta como também das técnicas de análise dos dados (FRANCO, 2005).

Independentemente de se optar por uma pesquisa que seguirá o método qualitativo, quantitativo ou mesmo quali-quantitativo, tem o pesquisador que se encontrar apto a saber avaliar os resultados oriundos da sua escolha.

Assim, a Análise de Conteúdo é uma das técnicas de análise de dados que tem sua origem mais remota ligada às tentativas iniciais dos homens de buscarem interpretar os livros sagrados e outros códigos de comunicação.

Como muito bem enuncia Bardin (2016, p.20): 
PALMEIRA, Lana Lisiêr de Lima; CORDEIRO, Carla Priscilla Barbosa Santos; PRADO, Edna Cristina do. A análise de conteúdo e sua importância como instrumento de interpretação dos dados qualitativos nas pesquisas educacionais

\begin{abstract}
A Hermenêutica, arte de interpretar os textos sagrados ou misteriosos, é uma prática muito antiga. O que é passível de interpretação? Mensagens obscuras que exigem uma interpretação, mensagens com um duplo sentido cuja significação profunda (a que importa aqui) só pode surgir depois de uma observação cuidadosa ou de uma intuição carismática.

Por detrás do discurso aparentemente geralmente simbólico e polissêmico esconde-se um sentido que convém desvendar. A interpretação dos sonhos, antiga ou moderna, a exegese religiosa (em especial a Bíblia), a explicação crítica de certos textos literários, até mesmo de práticas tão diferentes como a astrologia ou a psicanálise relevam de um processo hermenêutico[...]

A atitude interpretativa continua, em parte, a existir na análise de conteúdo, mas, é sustentada por processos técnicos de validação. Certos estudos assemelhavam-se pelo seu objeto à retórica (a propaganda, por exemplo), ou à lógica pelo seu procedimento (por exemplo, a análise de um desenvolvimento normativo de suas regras de enunciação), ou até mesmo pelo seu objetivo (a análise de conteúdo não é, esperamo-lo, nem doutrinal nem normativa).
\end{abstract}

A Análise de Conteúdo desenvolveu-se pioneiramente nos Estados Unidos, tendo em Lasswell um nome representativo no início de sua trajetória. Apenas em 1977, com a publicação da obra de Bardin, "Analyse de Contenu”, surge uma definição mais precisa sobre essa técnica, onde o método ganhou contornos que são os guias orientadores do estudo nos dias atuais.

De acordo com Bardin, o termo análise de conteúdo consiste em:

um conjunto de técnicas de análise das comunicações visando a obter, por procedimentos sistemáticos e objetivos de descrição do conteúdo das mensagens, indicadores (quantitativos ou não) que permitam a inferência de conhecimentos relativos às condições de produção/recepção (variáveis inferidas) destas mensagens (2016, p. 48).

Franco (2005), por sua vez, assevera que a análise de conteúdo é um método (para alguns uma técnica) utilizado para compreensão de significações em uma mensagem (que pode ser uma comunicação oral ou escrita, pode ser gestual, figurativa, etc.), que busca desvendar tanto o significado aparente quanto aquele aparentemente não percebido na fala de um interlocutor.

Nesse segmento de ideias, após lançar a conceituação mais ampla acerca da Análise de Conteúdo, convém, antes de avançar nas suas peculiaridades, fazer a distinção entre Análise de Conteúdo e Análise de Discurso, eis que apesar das duas técnicas beberem nas mesmas fontes, elas apresentam limites tênues e sutis, o que faz com que muitos não compreendam a diferença existente (FRANCO, 2005).

A Análise do Discurso intenciona observar os elementos presentes no momento de construção dos enunciados, sejam eles verbais ou não verbais.

De acordo com Pêcheux e sua corrente de linha francesa, deve-se olhar no discurso a relação que se estabelece entre língua/sujeito/história ou língua/ideologia. (2002, p. 87).

Assim, a linguagem não é estudada meramente como forma linguística, mas também como forma material da ideologia. 
Já a Análise de Conteúdo, conforme já visto em linhas anteriores, é um conjunto de técnicas de análise das comunicações que objetiva a inferência de conhecimentos pertinentes às condições de produção e recepção das mensagens enunciadas.

$\mathrm{Na}$ análise de conteúdo é muito importante distinguir o significado do sentido de determinados objetos do discurso. O significado é a definição a partir de "características definidoras", a partir do "corpus de significação". O sentido, por outro lado, é a "atribuição de um significado pessoal objetivado, que se concretiza na prática social e que se manifesta a partir das Representações Sociais, cognitivas, valorativas e emocionais, necessariamente contextualizadas" (FRANCO, 2005, p. 15).

Ainda na perspectiva de Franco,

\begin{abstract}
Aparentemente a linguística e a análise de conteúdo têm o mesmo objeto: a linguagem. Em verdade, porém, a distinção fundamental proposta por F. de Saussure, que fundou a linguística, entre língua e palavra marca a diferença: o objeto da linguística é a língua, quer dizer, o aspecto coletivo e virtual da linguagem, enquanto que o da análise do conteúdo é a palavra, isto é, o aspecto individual e atual (em ato) da linguagem. [...] A linguística estuda a língua para descrever o seu funcionamento. A análise de conteúdo procura conhecer aquilo que está por trás das palavras sobre as quais se debruça (2005, p.10).
\end{abstract}

Dessa forma, pode-se deduzir que a grande diferença entre as formas de análise em discussão repousa na questão de que a Análise do Discurso busca priorizar as condições de produção do corpus, enquanto a Análise do Conteúdo abarca, tanto a materialidade corporificada no texto, quanto as condições empíricas que deram origem ao mesmo, tentando captar seu sentido real na forma mais ampla possível.

Em outras palavras, pode-se afirmar que, diferentemente do que muitos pensam, essas duas técnicas não se conflitam. Ao contrário, elas vão ao encontro uma da outra complementando-se sempre que necessário.

Explicada a origem evolutiva da Análise de Conteúdo, esboçada sua definição e a diferenciação desta técnica para a Análise de Discurso, fulcral passar a trabalhar as fases em que se baseia a Análise de Conteúdo, de acordo com o que será realizado adiante.

\title{
As três fases da análise de conteúdo e sua importância nas pesquisas educacionais
}

Bardin (2016) anuncia que a utilização da análise de conteúdo prevê três fases, a saber: a) pré-análise; b) exploração do material; c) tratamento dos resultados (a inferência e a interpretação).

A primeira fase, a pré-análise, pode ser visualizada como uma etapa de organização, onde se estabelece um mecanismo de trabalho que, mesmo devendo ser preciso, com procedimentos bem definidos, pode ser flexível (BARDIN, 2016). 
Tal fase, segundo Bardin (2016), inclui a leitura "flutuante", ou seja, um contato preliminar com os documentos que serão analisados, a escolha dos mesmos, a formulação das hipóteses e objetivos, a criação dos indicadores que orientarão a interpretação, bem como a preparação de todo o material.

Dá-se, assim, início ao trabalho selecionando os documentos objeto de análise. Em se utilizando de entrevistas, deve-se fazer as devidas transcrições e seu conjunto representará o corpus da pesquisa.

Todavia, importante observar as regras de exaustividade (consiste em esgotar a totalidade da comunicação), representatividade (a amostra deve representar todo universo pesquisado); homogeneidade (os dados devem guardar relação com um mesmo assunto e serem obtidos por técnicas semelhantes); pertinência (os documentos devem se vincular ao conteúdo da pesquisa) e exclusividade (um elemento não deve ser classificado em mais de uma categoria).

Na segunda fase, de exploração do material, serão definidas as unidades de codificação, de acordo com os seguintes procedimentos de codificação: a escolha de unidades de registro, seleção de regras de contagem, a escolha de categorias (unidades de registro) e categorização.

Escolhida a unidade de codificação, far-se-á a classificação em blocos que demonstrem determinadas categorias, aptos a confirmar ou alterar aquelas, constantes nas hipóteses, e referenciais teóricos definidos a priori.

Posteriormente, agrupam-se os temas nas categorias definidas, em quadros matriciais, pelos pressupostos utilizados por Bardin (2016). Assim, após elaboração das categorias-síntese, passa-se à criação de cada categoria.

A definição pode seguir o conceito apresentado no referencial teórico ou ser baseada nas verbalizações relativas aos temas, ambos, título e definição, devendo ser registrados nos quadros matriciais. Ainda de acordo com Bardin (2016), as categorias podem ser criadas a priori ou a posteriori, isto é, a partir apenas da teoria ou após a coleta de dados.

Depois da construção das categorias, o pesquisador está a um passo da construção do conhecimento objetivado. Seguir os parâmetros deste método não significa, necessariamente, que o pesquisador conseguirá produzir um bom trabalho, pois todo esse processo não vale de nada sem a sua atuação criativa e coerente no momento da concatenação lógica das partes obtidas. Todavia, é, sem dúvida, um método que facilita imensamente todo o trabalho, além de criar parâmetros mais racionais e inteligentes de compreensão dos conteúdos de uma pesquisa.

A terceira fase do processo de análise do conteúdo é destinada ao tratamento dos resultados - a inferência e interpretação. Baseado nos resultados ainda brutos, o pesquisador terá a tarefa de buscar a sua real significação. 
Esta interpretação deverá ir além do conteúdo que apareceu nos documentos, já que o mais importante é o conteúdo latente, ou seja, o sentido que se encontra por trás do resultado aparentemente encontrado. (BARDIN, 2016).

A inferência na análise de conteúdo se guia por vários polos de atenção, que são os polos de atração da comunicação. É um instrumento de indução (roteiro de entrevistas) para se investigarem as causas (variáveis inferidas) a partir dos efeitos (variáveis de inferência ou indicadores, referências), segundo Bardin (2016, p. 138).

Em seguida, inicia-se a interpretação de conceitos. Aqui, deve-se ressaltar que os conceitos dão um sentido de referência geral, criando imagem significativa.

Os conceitos são oriundos da cultura estudada e da linguagem dos informantes. Assim, ao se desvendar um tema nos dados coletados, é preciso comparar enunciados e ações entre si, para ver se existe um conceito que os unifique. Quando se encontram temas diferentes, é necessário buscar similitudes que possa haver entre eles.

Não se pode deslembrar que durante a interpretação dos dados, é necessário olhar com atenção os marcos teóricos, ligados à investigação, já que eles sedimentam as vertentes da pesquisa, pois o elo entre os dados coletados e a fundamentação teórica é que dará sentido à interpretação do estudo.

Desse modo, finaliza-se o processo de Análise de Conteúdo, ficando evidente que a referida técnica é um caminho extremamente útil aos pesquisadores dos mais diversos campos do conhecimento, em especial nas pesquisas educacionais, onde se busca constantemente a construção de conhecimentos científicos.

Vê-se, assim, que a análise de conteúdo não é uma técnica realizada em momento único e isolado, sendo, antes de tudo, fruto de uma necessidade histórica e cultural de se encontrar as significações nem sempre claras aos intérpretes.

É uma forma de chegar ao ponto central de uma mensagem que utiliza uma série de procedimentos e fases de extrema relevância ao pesquisador, representando, sem dúvida, um marco no desenvolvimento dos métodos de análises nas ciências de forma geral.

\section{Resultados e discussão}

Metodologia da análise dos dados

Para compreender a importância da análise de conteúdo nas pesquisas educacionais, realizou-se uma pesquisa exploratória sobre a temática. Tornou-se necessário, para isto, uma revisão sistemática sobre estudos que utilizaram o método para de Análise de Conteúdo na área 
educacional nos últimos 5 anos, a fim de demonstrar como essa ferramenta pode ser útil em processos de análises de dados.

Para isto, optou-se pela realização de uma pesquisa exploratória, ampliando-se a familiarização com o problema (GIL, 2008). Por sua vez, os dados foram colhidos por meio de um levantamento bibliográfico. Nas palavras de Bandeira (2020, p. 3), "a pesquisa de levantamento ou "Survey" visa descrever a distribuição das características ou de fenômenos que ocorrem naturalmente em grupos da população”. Este tipo de pesquisa acaba por medir variáveis descritivas dos sujeitos de uma pesquisa. O levantamento bibliográfico, por seu turno, se volta ao

\section{[...] mapeamento da informação produzida e publicada pela humanidade, bem como, com a localização, organização, ampla disseminação e preservação da produção intelectual - instituições como bibliotecas, centros de documentação e de informação, públicos ou privados, e empresas dedicadas exclusivamente à gestão do conhecimento e informação, mapearam e mapeiam o que é produzido e publicado (GALVÃO, 2006, p. 1).}

Neste sentido, realizou-se um levantamento sobre a produção intelectual de pesquisas que realizaram a análise de conteúdo na esfera educacional como método de análise de dados, adotando-se como recorte temporal o período entre 2015 e 2019.

As bases de dados selecionadas para o levantamento foram Scielo, Portal da Capes de periódicos e BDTD. Optou-se por excluir o Google Acadêmico das bases analisadas porque a plataforma não possui filtros suficientes em sua busca avançada, como, por exemplo, a escolha dos tipos de trabalhos acadêmicos (nesta pesquisa priorizaram-se os artigos, dissertações e teses), o que geraria resultados muito abrangentes.

Para fins de compreensão do uso da análise de conteúdo na área educacional, foram realizadas duas buscas em cada base de dados: uma voltada à utilização da análise de conteúdo enquanto instrumento de pesquisa para análise de dados de forma abrangente (em todas as áreas do conhecimento) e outra voltada apenas para a área educacional. Seguindo-se este percurso, foram colhidos os dados que seguem.

\section{A utilização da análise de conteúdo em pesquisas educacionais}

Enquanto método de análise de dados, a análise de conteúdo tem se revelado grande aliada das pesquisas qualitativas, no Brasil. Neste sentido, realizou-se um levantamento bibliográfico com o objetivo de quantificar a evolução da análise de conteúdo nas pesquisas educacionais publicadas em periódicos da área educacional, dissertações e teses. Para quantificar seu uso, foram analisadas as pesquisas publicadas na Scielo, Portal de Periódicos da Capes e BDTD entre 2015 e 2019. 
Na primeira plataforma analisada, a Scielo, a análise de conteúdo foi utilizada em 240 artigos no período mencionado em todas as áreas do conhecimento, sendo que desse total 35 pesquisas pertenciam à área da Educação, conforme pode ser visto no quadro abaixo:

Quadro 1 - Artigos científicos que utilizaram a análise de conteúdo publicados na Scielo

\begin{tabular}{|c|c|c|}
\hline & $\begin{array}{c}\text { Busca } 1 \text { - pesquisas na área da } \\
\text { educação }\end{array}$ & $\begin{array}{c}\text { Busca } 2 \text { - pesquisas em } \\
\text { todas as áreas }\end{array}$ \\
\hline Tipo de consulta à plataforma & busca avançada & busca avançada \\
\hline Termos de busca & "análise de conteúdo" + "Bardin" & $\begin{array}{l}\text { "análise de conteúdo" + } \\
\text { "Bardin" }\end{array}$ \\
\hline Idioma & Português & Português \\
\hline Ano de publicação & 2015, 2016, 2017, 2018, 2019 & 2015, 2016, 2017, 2018, 2019 \\
\hline Área temática principal & Ciências Humanas & Todas \\
\hline Área temática secundária & Educação e Pesquisa Educacional & Todas \\
\hline Resultado & 35 & 240 \\
\hline
\end{tabular}

Fonte: Scielo, 2020a; 2020b.

Outro dado interessante que pôde ser percebido foi o aumento da utilização do método na área educacional ao longo dos últimos anos. Em relação aos artigos publicados na plataforma Scielo, o gráfico abaixo revela uma crescente utilização da mesma em pesquisas educacionais entre 2015 e 2018, e um decréscimo no ano de 2019:

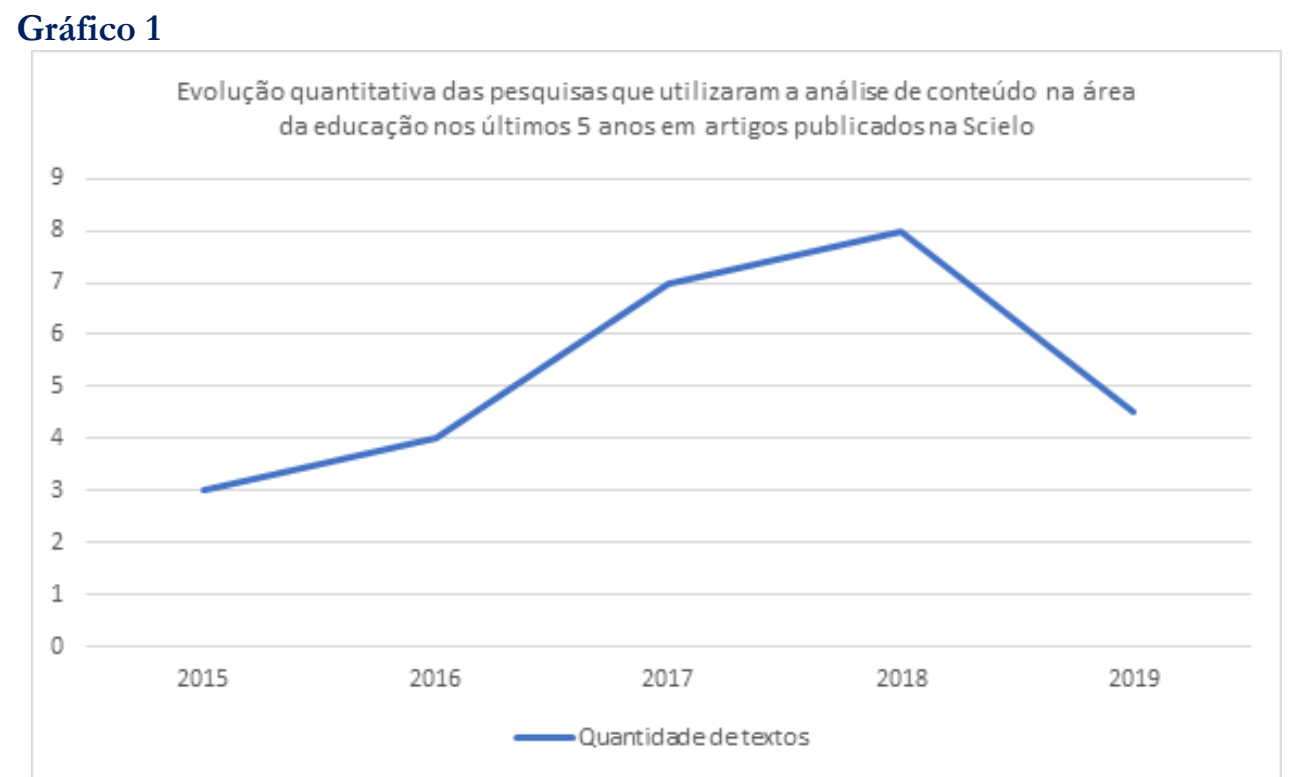

Fonte: Scielo, 2020b.

A outra base de dados consultada foi o Portal de Periódicos da Capes. A consulta realizada nesta base revelou 549 pesquisas que utilizaram a análise de conteúdo enquanto método de análise de dados, das quais 161 pertenciam à área da Educação: 
PALMEIRA, Lana Lisiêr de Lima; CORDEIRO, Carla Priscilla Barbosa Santos; PRADO, Edna Cristina do. A análise de conteúdo e sua importância como instrumento de interpretação dos dados qualitativos nas pesquisas educacionais

Quadro 2 - Artigos científicos que utilizaram a análise de conteúdo publicados no Portal de Periódicos da Capes

\begin{tabular}{|l|l|l|}
\hline & \multicolumn{1}{|c|}{$\begin{array}{c}\text { Busca 1 - pesquisas na área da } \\
\text { educação }\end{array}$} & $\begin{array}{c}\text { Busca 2 - pesquisas em todas as } \\
\text { áreas }\end{array}$ \\
\hline Tipo de consulta à plataforma & busca avançada & busca avançada \\
\hline Termos de busca & "análise de conteúdo" + "Bardin" & "análise de conteúdo" + "Bardin" \\
\hline Idioma & Português & Português \\
\hline Ano de publicação & $2015,2016,2017,2018,2019$ & $2015,2016,2017,2018,2019$ \\
\hline Tópico (área) & $\begin{array}{l}\text { Educação e pesquisa educacional } \\
\text { (Education \& Educational Research); } \\
\text { pesquisa qualitativa (Qualitative } \\
\text { research); educação (education). }\end{array}$ & Todas \\
\hline Resultado & \multicolumn{1}{|c|}{$\mathbf{1 6 1}$} & $\mathbf{5 4 9}$ \\
\hline
\end{tabular}

Fonte: CAPES, 2020a; 2020b.

Este é um resultado bastante expressivo da importância que a análise de conteúdo assume na esfera educacional, pois quase $1 / 3$ de todas as pesquisas que utilizaram o método eram desta área (29,32\%) (CAPES, 2020b). Em relação à evolução quantitativa do uso da técnica nas pesquisas educacionais, observou-se que houve um decréscimo de seu uso de 2016 em diante, como pode ser visto no gráfico abaixo:

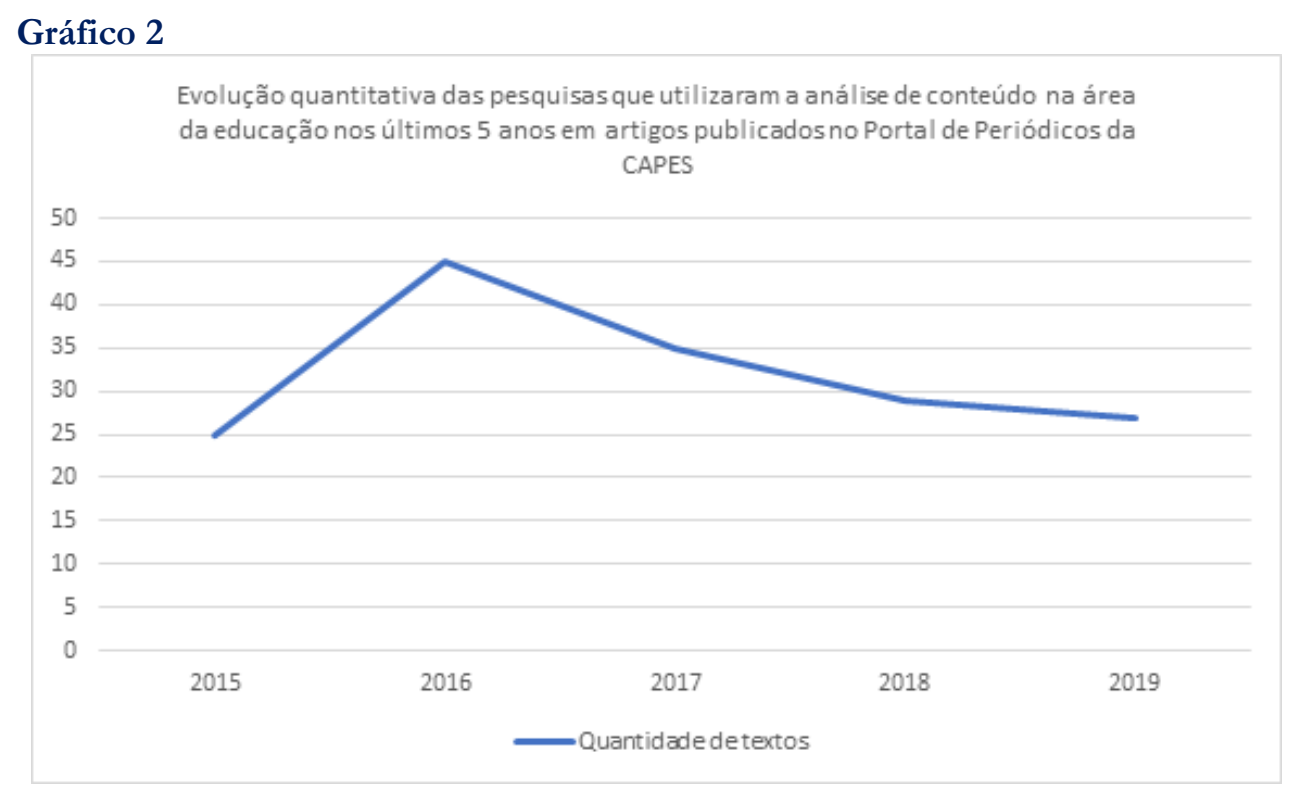

Fonte: Portal de Periódicos da Capes, 2020b.

Também foi possível avaliar as pesquisas provenientes dos programas de pós-graduação stricto sensu no país, tanto na área educacional quanto nas demais áreas: 
Quadro 3 - Dissertações e teses que utilizaram a análise de conteúdo publicados no Portal de Periódicos da Capes

\begin{tabular}{|l|l|l|}
\hline & $\begin{array}{c}\text { Busca 1 - pesquisas na área da } \\
\text { educação }\end{array}$ & \multicolumn{1}{|c|}{$\begin{array}{c}\text { Busca 2 - pesquisas em todas as } \\
\text { áreas }\end{array}$} \\
\hline Tipo de consulta à plataforma & busca avançada & busca avançada \\
\hline Termos de busca & "análise de conteúdo" + "Bardin" & "análise de conteúdo" + "Bardin" \\
\hline Idioma & Português & Português \\
\hline Ano de publicação & $2015,2016,2017,2018,2019$ & $2015,2016,2017,2018,2019$ \\
\hline Programas & $\begin{array}{l}\text { Pós-Graduação Stricto em } \\
\text { Educação }\end{array}$ & Todos \\
\hline Resultado & \multicolumn{1}{c|}{$\mathbf{1 5 2}$} & $\mathbf{2 . 0 3 3}$ \\
\hline
\end{tabular}

Fonte: BDTD, 2020a; 2020b.

Proporcionalmente, as dissertações e teses que utilizaram a análise de conteúdo para interpretação dos dados na área da educação, em comparação com as outras áreas, foram publicadas em quantidade bem menor que os artigos publicados nas duas bases de dados anteriores (7,47\% das dissertações e teses utilizaram a análise de conteúdo nas pesquisas educacionais) (BDTD, 2020b).

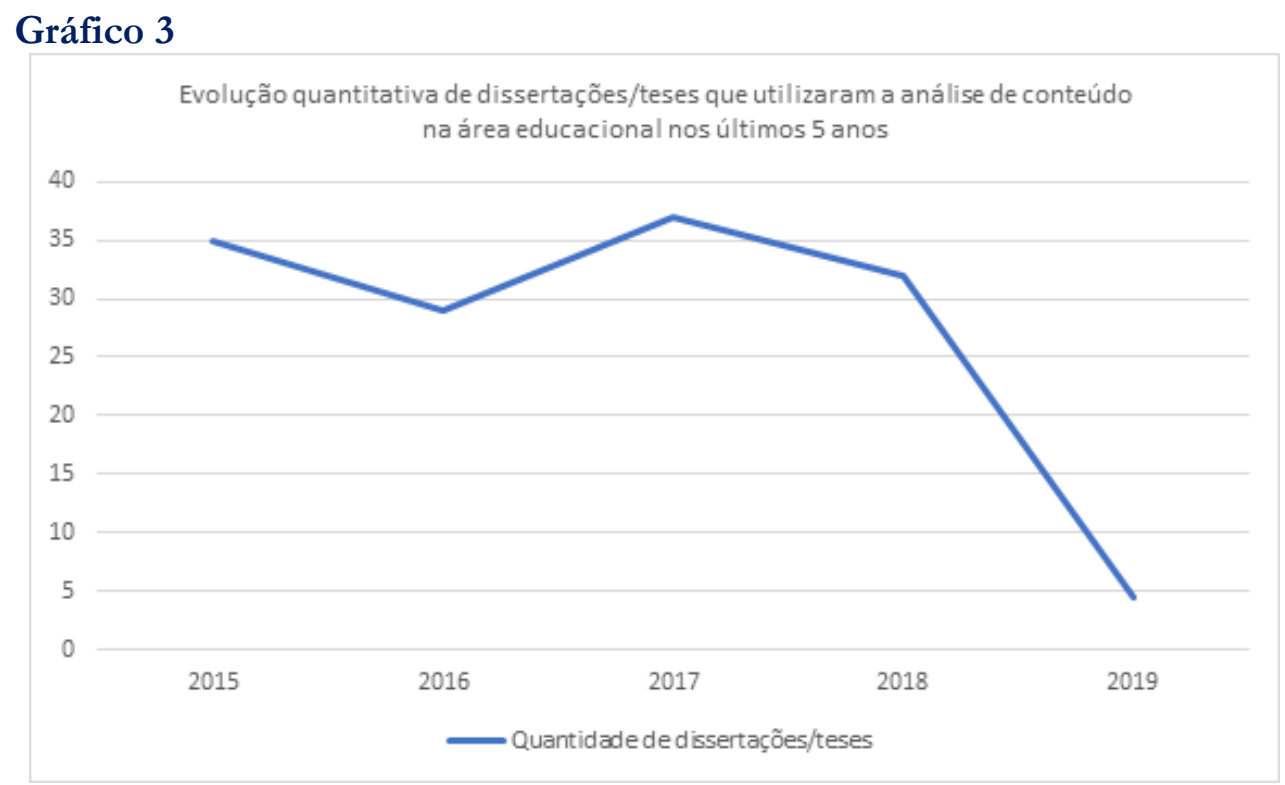

Fonte: BDTD, 2020b.

Embora não tenha ocorrido nenhum aumento significativo na publicação de dissertações e teses na área da educação no período analisado, o gráfico revela um decréscimo acentuado nas pesquisas também no ano de 2019.

\section{Conclusão}

O presente trabalho teve como intuito fazer uma discussão em torno dos elementos basilares da Análise de Conteúdo, realizando, de início, um breve resgaste histórico da forma pela 
qual a ciência se corporificou no mundo da pesquisa, chegando-se ao objeto central do estudo, com a apresentação dos aspectos mais característicos dessa técnica de análise de dados.

Por saber que os traços que distinguem a Análise de Conteúdo da Análise do Discurso não são ainda tão claros para muitos que iniciam as leituras nesse campo, ressaltou-se, ainda que rapidamente, o que vem a representar cada uma dessas técnicas com sua diferença primordial, tentando melhor situar as ideias até então declinadas.

Em seguida, buscou-se evidenciar as fases metodológicas que embasam a Análise de Conteúdo, desde a pré-análise até o tratamento dos dados coletados, onde se trabalhou a inferência e a interpretação, a fim de demonstrar a importância e riqueza dessa técnica, a qual se apresenta como ferramenta extremamente útil não só à elucidação de sentidos, muitas vezes latentes nas mensagens, mas também como forte mecanismo de interpretação das percepções dos atores sociais envolvidos em determinada conjuntura de pesquisa.

A análise de conteúdo se mostrou uma importante técnica na pesquisa educacional, em especial em análises qualitativas, como pode ser percebido do levantamento bibliográfico realizado nas bases de dados Scielo, Portal de Periódicos da Capes e BDTD. Entre os anos de 2015 e 2019 a análise de conteúdo foi bastante utilizada em proporção muito maior na área da educação do que em outras áreas, em todas as plataformas utilizadas. No entanto, algumas observações não poderiam escapar a esta análise.

Um dado muito significativo que pôde ser extraído dos dados da plataforma Scielo foi o aumento das publicações na área educacional, entre 2015 e 2018, que utilizaram a análise de conteúdo como técnica de análise de dados. No entanto, houve um decréscimo nas publicações em 2019, como pode ser visto no gráfico 1. Embora não haja uma pesquisa formal sobre os fatores que podem ser dados como causa do decréscimo de publicações em 2019, não se pode desconhecer o desmonte na educação superior que tem ocorrido ao longo do último ano, efeito direto das recentes políticas governamentais de corte e contingenciamento de gastos em pesquisa nas universidades, conforme amplamente noticiado na mídia brasileira (FOLHA DE SÃO PAULO, 2019). Esta é uma das principais causas dessa diminuição na quantidade de pesquisas em todas as áreas ao longo de 2019.

Os dados extraídos do Portal da Capes de Periódicos também revelaram, por sua vez, o decréscimo do uso do método em pesquisas educacionais, a partir de 2017. Esse resultado reforça a hipótese acima esboçada, diante dos dados da plataforma Scielo, de que a diminuição dos investimentos em pesquisa nas universidades, enquanto política de governo, afetou a produção de pesquisas científicas na área da Educação. 
Igualmente, a análise das dissertações e teses publicadas na BDTD revela que a análise de conteúdo também é uma técnica importante enquanto aliada das pesquisas educacionais, o que pode ser percebido pela quantidade significativa de pesquisas publicadas neste sentido. Percebeuse também uma queda acentuada nas pesquisas que utilizaram a técnica em 2019. Trata-se, sem dúvida, de um dado significativo que revela uma preocupante realidade da pesquisa científica no país, que precisa ser discutida e avaliada (refere-se à "preocupante realidade") sobre o prisma das orientações de governo para a educação.

Assim, à guisa de conclusão, entende-se que é nítida a percepção de que a Análise de Conteúdo está para além de uma mera técnica, representando uma conquista metodológica de grande complexidade para todos os pesquisadores que buscam desbravar horizontes ainda circundados pelas mais variadas codificações dos dados comunicativos.

No entanto, sem investimento em pesquisa na área educacional, diga-se, sem os recursos necessários para pesquisadores da graduação, mestrado e doutorado, as pesquisas empíricas deixam de ser realizadas, ante a ausência de recursos materiais para sua realização. Sem investimento em pesquisa, estudos voltados à realidade, à resolução de problemas da educação atual, e de todos os outros tipos, ficam estagnados, e o país deixa de avançar no sentido da resolução de seus problemas sociais.

\section{Referências}

ABBAGNANO, Nicola. Dicionário de Filosofia. São Paulo: Martins Fontes, 2000.

ARENDT, Hannah. A Condição Humana. Rio de Janeiro: Forense Universitária, 2016.

BDTD. Busca avançada. Disponível em:

http://bdtd.ibict.br/vufind/Search/Results?lookfor $=$ an $\% \mathrm{C} 3 \%$ A1lise + de + conte $\% C 3 \%$ BAdo+ $\% 2 \mathrm{~B}+$ bardin\&type $=$ AllFields\&daterange $\% 5 \mathrm{~B} \% 5 \mathrm{D}=$ publishDate\&publishDatefrom $=2015 \& \mathrm{publ}$ ishDateto=2019. Acesso em: 01.06.2020a.

BDTD. Busca avancada. Disponível em: http://bdtd.ibict.br/vufind/Search/Results?filter $\% 5 \mathrm{~B} \% 5 \mathrm{D}=$ dc.publisher.program.fl_str_mv $\% 3$ $\mathrm{A} \% 22$ Programa $+\mathrm{de}+\mathrm{P} \% \mathrm{C} 3 \% \mathrm{~B} 3 \mathrm{~s}-$

Gradua $\% \mathrm{C} 3 \% \mathrm{~A} 7 \% \mathrm{C} 3 \% \mathrm{~A} 3 \mathrm{o}+\mathrm{em}+\mathrm{Educa} \% \mathrm{C} 3 \% \mathrm{~A} 7 \% \mathrm{C} 3 \% \mathrm{~A} 3 \mathrm{0} \% 22 \&$ filter $\% 5 \mathrm{~B} \% 5 \mathrm{D}=$ language $\%$ $3 \mathrm{~A} \% 22$ por $\% 22 \&$ lookfor $=$ an $\% \mathrm{C} 3 \%$ A 1 lise + de + conte $\% \mathrm{C} 3 \% \mathrm{BAdo}+\% 2 \mathrm{~B}+$ bardin\&type $=$ AllField s\&daterange $\% 5 \mathrm{~B} \% 5 \mathrm{D}=$ publishDate\&publishDatefrom $=2015 \&$ publishDateto $=2019$. Acesso em: 01.06.2020b.

BANDEIRA, Marina. Tipos de Pesquisa. Disponível em: https://ufsj.edu.br/portalrepositorio/File/lapsam/Metodo $\% 20 \mathrm{de}^{2} \% 20$ pesquisa/Metodos $\% 20 \mathrm{de} \% 20$ pesquisa $\% 202013 / \mathrm{Te}$ xto_1B_-_Tipos_de_Pesquisa.pdf. Acesso em 01.05.2020.

BARDIN, Laurence. Análise de Conteúdo. São Paulo: Edições 70, 2016. 
BAUER, M.W. \& GASKELL, G. (org.) Pesquisa Qualitativa com Texto, Imagem e Som - um manual prático. 2 ed. Petrópolis: Vozes, 2003.

FOLHA DE SÃO PAULO. Gestão Bolsonaro far corte generalizado em bolsas de pesquisa no país. Disponível em: https://www1.folha.uol.com.br/educacao/2019/05/gestao-bolsonaro-faz-cortegeneralizado-em-bolsas-de-pesquisa-pelo-pais.shtml. Acesso em 01.05.2020.

FRANCO, Maria Laura Puglisi Barbosa. Análise de Conteúdo. 2.ed. Brasília: Liber Livro, 2005.

GADAMER, Hans-Georg. Verdade e Método. Petrópolis: Vozes, 1997.

GALVÃO, Maria Cristiane Barbosa. Levantamento bibliográfico e a pesquisa científica. Disponível em: http://www2.eerp.usp.br/Nepien/DisponibilizarArquivos/Levantamento_bibliografico_Cristian eGalv.pdf. Acesso em 01.05.2020.

GIL, Antonio Carlos. Como elaborar projetos de pesquisa. 4. ed. São Paulo: Atlas, 2008.

GUERRA, Isabel Carvalho. Pesquisa Qualitativa e Análise de Conteúdo. Cascais: Princípia, 2006.

HEIDEGGER, Martin. Ser e Tempo. Petrópolis: Vozes, 2014.

KUHN, Thomas (1962). A estrutura das revolucões científicas. 5 ed. São Paulo: Editora Perspectiva, 1997.

MORAES, Roque. Análise de conteúdo. Revista Educação. Porto Alegre, v. 22, n. 37, p. 7-32, 1999.

ORLANDI, Eni. Análise de Discurso: princípios e procedimentos. Campinas (SP): Pontes, 1999.

PÊCHEUX, Michel. O Discurso: estrutura ou acontecimento. $3^{\mathrm{a}}$ ed. Campinas (SP): Pontes, 2002.

POPPER, K. Lógica da pesquisa cientifica. São Paulo: EDUSP, 1985.

PORTAL DA CAPES. Busca avançada. Disponível em:

https:/ /www.periodicos.capes.gov.br/ ?option $=$ com_pmetabusca\&mn $=88 \& s m n=88 \& t y p e=m \&$ metalib=aHR0cHM6Ly9ybnAtcHJpbW8uaG9zdGVkLmV4bGlicmlzZ3JvdXAuY29tL3ByaW1v X2xpYnJhenkvbGlid2ViL2FjdGlvbi9zZWFyY2guZG8\%2FZHNjbnQ9MCZwY0F2YWlsYWJp bHR5TW9kZT1mYWxzZSZmcmJnPSZzY3Auc2Nwcz1wcmltb19jZW50cmFsX211bHRpcGxl X2ZlJnRhYj1kZWZhdWx0X3RhYiZjdD1zZWFyY2gmbW9kZT1CYXNpYyZkdW09dHJ1ZSZ pbmR4PTEmZm49c2VhcmNoJnZpZD1DQVBFU19WMQ\%3D\%3D\&buscaRapidaTermo=+ $\% \mathrm{E} 2 \% 80 \%$ Can $\% \mathrm{C} 3 \%$ A 1 lise + de + conte $\% \mathrm{C} 3 \% \mathrm{BAdo} \% \mathrm{E} 2 \% 80 \% 9 \mathrm{D}+\% 2 \mathrm{~B}+\% \mathrm{E} 2 \% 80 \% 9 \mathrm{CB}$ ard in $\% \mathrm{E} 2 \% 80 \% 9 \mathrm{D}$. Acesso em: 01.06.2020a.

PORTAL DA CAPES. Busca avançada. Disponível em:

https: $/ /$ www.periodicos.capes.gov.br/?option $=$ com_pmetabusca\&mn $=88 \& s m n=88 \&$ type $=\mathrm{m} \&$ metalib=aHR0cHM6Ly9ybnAtcHJpbW8uaG9zdGVkLmV4bGlicmlzZ3JvdXAuY29tL3ByaW1v X2xpYnJhenkvbGlid2ViL2FjdGlvbi9zZWFyY2guZG8\%2FZHNjbnQ9MCZwY0F2YWlsYWJp bHR5TW9kZT1mYWxzZSZmcmJnPSZzY3Auc2Nwcz1wcmltb19jZW50cmFsX211bHRpcGxl X2ZIJnRhYj1kZWZhdWx0X3RhYiZjdD1zZWFyY2gmbW9kZT1CYXNpYyZkdW09dHJ1ZSZ pbmR4PTEmZm49c2VhcmNoJnZpZD1DQVBFU19WMQ\%3D\%3D\&buscaRapidaTermo=+ $\% \mathrm{E} 2 \% 80 \%$ Can $\% \mathrm{C} 3 \%$ A 1 lise + de + conte $\% \mathrm{C} 3 \% \mathrm{BAdo} \% \mathrm{E} 2 \% 80 \% 9 \mathrm{D}+\% 2 \mathrm{~B}+\% \mathrm{E} 2 \% 80 \% 9 \mathrm{CB}$ ard in $\% \mathrm{E} 2 \% 80 \% 9 \mathrm{D}$. Acesso em: 01.06.2020b. 
REALE, Giovanni. História da filosofia: De Spinoza a Kant. São Paulo: Paulus, 2003.

SCIELO. Busca avançada. Disponível em:

https://search.scielo.org $/$ fb $=\& q=\% \mathrm{E} 2 \% 80 \% 9$ Can $\% \mathrm{C} 3 \%$ A 1 lise + de + conte $\% \mathrm{C} 3 \% \mathrm{BAddo} \% \mathrm{E} 2 \%$ $80 \% 9 \mathrm{D}+\% 2 \mathrm{~B}+\% \mathrm{E} 2 \% 80 \% 9 \mathrm{CB}$ ardin $\% \mathrm{E} 2 \% 80 \% 9 \mathrm{D} \&$ lang $=$ pt\&count $=15 \&$ from $=1 \&$ output $=$ site $\&$ sort $=\&$ format $=$ summary\&page $=1 \&$ where $=\&$ filter $\% 5$ Byear_cluster $\% 5 \mathrm{D} \% 5 \mathrm{~B} \% 5 \mathrm{D}=2019 \&$ filte $\mathrm{r} \% 5$ Byear_cluster $\% 5 \mathrm{D} \% 5 \mathrm{~B} \% 5 \mathrm{D}=2018 \&$ filter $\% 5$ Byear_cluster $\% 5 \mathrm{D} \% 5 \mathrm{~B} \% 5 \mathrm{D}=2016 \&$ filter $\% 5 \mathrm{~B}$ year_cluster $\% 5 \mathrm{D} \% 5 \mathrm{~B} \% 5 \mathrm{D}=2017 \&$ filter $\% 5$ Byear_cluster $\% 5 \mathrm{D} \% 5 \mathrm{~B} \% 5 \mathrm{D}=2015$. Acesso em: 01.06.2020a.

SCIELO. Busca avançada. Disponível em:

https: / / search.scielo.org $/$ ?q $=\% \mathrm{E} 2 \% 80 \% 9 \mathrm{Can} \% \mathrm{C} 3 \%$ A 1 lise + de + conte $\% \mathrm{C} 3 \% \mathrm{BAdo} \% \mathrm{E} 2 \% 80 \% 9$ $\mathrm{D}+\% 2 \mathrm{~B}+\% \mathrm{E} 2 \% 80 \% 9 \mathrm{CB}$ ardin $\% \mathrm{E} 2 \% 80 \% 9 \mathrm{D} \&$ lang $=$ pt\&count $=15 \&$ from $=31 \&$ output $=$ site $\&$ so $\mathrm{rt}=\&$ format $=$ summary\&fb $=\&$ page $=3 \&$ filter $\% 5 \mathrm{Bin} \% 5 \mathrm{D} \% 5 \mathrm{~B} \% 5 \mathrm{D}=$ scl\&filter $\% 5 \mathrm{Bla} \% 5 \mathrm{D} \% 5 \mathrm{~B} \%$ $5 \mathrm{D}=$ pt\&filter $\% 5$ Byear_cluster $\% 5 \mathrm{D} \% 5 \mathrm{~B} \% 5 \mathrm{D}=2019 \&$ filter $\% 5$ Byear_cluster $\% 5 \mathrm{D} \% 5 \mathrm{~B} \% 5 \mathrm{D}=201$ $8 \&$ filter $\% 5$ Byear_cluster $\% 5 \mathrm{D} \% 5 \mathrm{~B} \% 5 \mathrm{D}=2016 \&$ filter $\% 5$ Byear_cluster $\% 5 \mathrm{D} \% 5 \mathrm{~B} \% 5 \mathrm{D}=2017 \&$ filt er $\% 5$ Byear_cluster $\% 5 \mathrm{D} \% 5 \mathrm{~B} \% 5 \mathrm{D}=2015 \&$ filter $\% 5 \mathrm{~B}$ subject_area $\% 5 \mathrm{D} \% 5 \mathrm{~B} \% 5 \mathrm{D}=$ Human + Scien ces\&filter $\% 5$ Bwok_subject_categories $\% 5 \mathrm{D} \% 5 \mathrm{~B} \% 5 \mathrm{D}=$ education $+\% 26+$ educational + research. Acesso em: 01.06.2020b.

\section{Notas}

${ }^{1}$ Essencial esclarecer que se optou por trazer o termo também em inglês em razão da tradição empírica experimental ter origem profundamente inglesa, desde Locke, Francis Bacon, diferente do racionalismo que é uma corrente mais francesa muito trabalhada em Descartes.

2 O termo "Dasein" é uma expressão característica da Filosofia existencialista de Heidegger que significa o homem como "ser-ấ", ou seja, o homem lançado ao mundo. 